\title{
Molecular Localization of Frog Retinal Receptor Photopigment by Electron Microscopy and Low-angle X-ray Diffraction
}

\author{
J. K. Blasie†, C. R. Worthington \\ Department of Physics and Biophysics \\ Research Division, The University of Michigan, Ann Arbor, Mich., U.S.A. \\ AND \\ M. M. Dewey \\ Department of Anatomy \\ Woman's Medical College of Pennsylvania, Philadelphia, Pa., U.S.A.
}

(Received 8 May 1968, and in revised form 21 October 1968)

Low-angle X-ray diffraction patterns were obtained from ordered ultracentrifugal pellets of wet receptor disk membranes which had been either treated with antirhodopsin serum, normal rabbit serum, serum albumin, or untreated prior to sedimentation. A preliminary analysis of these patterns indicated: (a) differences between antirhodopsin serum treated and untreated preparations are due to conjugation of antirhodopsin molecules with their antigen in the disk membranes and not non-specific adsorption of other serum proteins to the disk membranes, (b) the planar ordering of the adsorbed antirhodopsin molecules over the surface of the disk membrane is nearly identical to that of the 40 to 50 A particles in the untreated disk membrane.

A detailed Fourier analysis of these patterns in terms of a planar liquid-like arrangement of the 40 to $50 \AA$ particles in the untreated disk membranes and the antirhodopsin molecules adsorbed to the antirhodopsin serum treated disk membranes confirmed our preliminary analysis. The planar liquid-like arrangement of the 40 to $50 \AA$ particles is nearly identical to that of the adsorbed antirhodopsin molecules $(3 \cdot 0$ and $3 \cdot 1$ nearest neighbors at a separation of 56 and $58 \AA$ respectively at $26^{\circ} \pm 0.2 \mathrm{deg}$.C). Thus, the 40 to $50 \AA$ particles of the wet untreated disk membranes are the photopigment molecules.

Electron micrographs of phosphotungstate negatively-stained disk membrane demonstrate particles $\sim 40 \AA$ in diameter within the disk membrane. Optical transforms of these electron micrographs show that these particles appearing in the micrograph are arranged in a planar square array with a unit cell side of $\sim 70 \AA$. Correlation of these results with those obtained by low-angle X-ray diffraction in ultracentrifugal pellets of phosphotungstate stained and dried disk membranes as well as on wet pellets of untreated disk membranes before, during and after drying indicates the following: the $\sim 40 \AA$ diameter particles seen in the electron micrographs are most, likely the same 40 to $50 \AA$ particles giving rise to the observed low-angle $\mathrm{X}$-ray diffraction from wet disk membranes. Hence the particles seen in the electron micrographs are most likely the nonpolar cores of the photopigment molecules.

$\dagger$ Present address: Biophysics Dept., University of Pennsylvania, Philadelphia, Pa., U.S.A. 


\section{Introduction}

In an earlier paper (Blasie, Dewey, Blaurock \& Worthington, 1965), the occurrence of particles 40 to $50 \AA$ in diameter in frog retinal receptor disk membranes was reported. This description followed from electron microscopic studies of negativelystained disk membranes and low-angle X-ray diffraction studies of partially dried ultracentrifugal pellets of the disk membranes. A criticism of this earlier work was that these particles might not exist in wet preparations. However, our X-ray diffraction results on "moisture-sealed" preparations and their interpretation described in that paper are consistent with the results from wet preparations to be described here. Hence, at the time of that earlier paper, low-angle X-ray diffraction from particles within wet disk membrane preparations had been observed although their composition and precise ordering had not been resolved. Two possibilities for the composition of the particles were given, either spherical lipid micelles which have been observed in electron micrographs of macromolecular lipid complexes (Lucy \& Glauert, 1964) and thought to occur in certain types of biological membranes after freeze drying (Sjöstrand, 1963), or the photopigment molecules of the disk membranes.

In these papers we describe further work which has led us to identify the particles of disk membranes as photopigment molecules and to define their planar ordering in the disk membrane. In particular, we have made use of an antibody preparation specific for rhodopsin of frog retinal receptors (Dewey, Blasie, Davis \& Barr, 1969 and preliminary reports by these authors mentioned in that paper). Our identification follows from: (a) electron microscopic studies of phosphotungstate negatively-stained disk membranes and previous low-angle X-ray diffraction studies (Blasie et al., 1965) on ultracentrifugal pellets of phosphotungstate stained and dried disk membranes and pellets of untreated dried disk membranes and (b) low-angle X-ray diffraction studies of ultracentrifugal pellets of untreated disk membranes or disk membranes treated previously with antirhodopsin immune serum or non-specific protein as a control. The X-ray diffraction patterns were obtained from fully hydrated disk membrane preparations. This work has been reported briefly elsewhere (Blasie, Dewey \& Worthington, 1966 Abstr. Ann. Meeting Histochemical Society, Atlantic City).

\section{Materials and Methods}

\section{(a) Electron microscopy}

Frog (Rana pipiens) retinal receptor disk membrane preparations were obtained by disrupting dark-adapted frog retinas in $45 \%$ sucrose (16 retinas $/ 7 \mathrm{ml} .45 \%$ sucrose) with a glass syringe and subsequent flotation to a $45 \%$ sucrose-0.1 m-phosphate buffer ( $\mathrm{pH} 7 \cdot 4$ ) interface at $25,000 \mathrm{~g}$ for $15 \mathrm{~min}$ in a Spinco $\mathrm{SW25} \cdot 1$ swinging bucket rotor at $4^{\circ} \mathrm{C}$. The receptor disk membranes were collected from the interface and negatively stained with $1 \%$ sodium phosphotungstate and then deposited on Formvar-coated copper grids and dried.

These negatively-stained preparations were then viewed with a Hitachi HU11A electron microscope using projector pole-piece no. 2 at range 4 , a compensated $20 \mu$ objective aperture, and magnifications between 20,000 and 100,000 at $50 \mathrm{kv}$.

Optical diffraction patterns were obtained directly from the electron micrographs using an optical transform apparatus (R. B. Pullen \& Co., London) with a He-Ne laser source (Spectra-Physics). The electron micrographs were printed on Kodalith Ortho film. The transforms were obtained from these prints which were mounted between two optical 
flats using immersion oil. The interparticle distance for the particles of the disk membranes was about $3 \mathrm{~mm}$ in such prints. The total area of the print used in obtaining the transform contained on the order of 1000 particles. Optical diffraction patterns were also obtained in the following way. Masks for the transforms were made by punching $\frac{1}{2} \mathrm{~mm}$ holes at the centers of the particles of tho disk mombranes as thoy appeared in prints (photographic paper) of the electron micrographs. Such masks contained at least 1000 holes and the center-to-center spacing of the holes was about $3 \mathrm{~mm}$. The optical transform apparatus was calibrated using a known lattice and hence the size and symmetry of the array of particles appearing in the electron micrograph print was obtained. Dimensions were assigned knowing the total magnification (electronic and photographic) of the print used.

Disk membrane preparations obtained from about 50 retinas in the manner described were sedimented in Ringers solution into a pellet at $68,000 \mathrm{~g}$ for $1 \mathrm{hr}$ at $4^{\circ} \mathrm{C}$. The pellet was fixed for $2 \mathrm{hr}$ in $6 \%$ glutaraldehyde in $0.085 \mathrm{M}$-phosphate buffer ( $\mathrm{pH} 7 \cdot 4$ ), washed, and post-fixed for $2 \mathrm{hr}$ in $1 \% \mathrm{OSO}_{4}$ in $0.028 \mathrm{~m}$-Veronal acetate buffer (pH 7.4), adjusted to $230 \mathrm{mOsm}$ with sucrose. The pellet was then dehydrated and embedded in Epon 812 . The pellet was sectioned on an LKB ultramicrotome parallel to the sedimentation axis and the sections were stained with uranyl acetate and lead hydroxide.

\section{(b) Low-angle $X$-ray diffraction}

Retinal receptor disk membranes of about 50 retinas were collected by centrifugation from a $45 \%$ sucrose-0.01 M-phosphate buffer interface as in the previous section. The untreated disk membranes were then taken up in about $15 \mathrm{ml}$. of Ringers solution and sedimented in a specially designed cell with a cylindrical cavity $3 \mathrm{~mm}$ in diameter sealed at the bottom by a Melinex window. The disk membranes were sedimented at $68,000 \mathrm{~g}$ for $1 \mathrm{hr}$ forming a cylindrical pellet 2 to $3 \mathrm{~mm}$ in thickness. The sedimentation cell was demounted and the part of the cell containing the disk membrane pellet was fitted tightly into an aluminum and brass temperalure chamber (Melinex windows) aligned such that the X-ray beam was incident along the axis of sedimentation. A $1 \mathrm{~mm}$ layer of Ringers solution remained over the surface of the disk membrane pellet and the cell was sealed in the temperature chamber keeping the pellet fully hydrated (wet) for as long as the chamber was sealed. The temperature chamber was cooled or heated via circulating fluids and the temperature could be maintained within \pm 0.2 deg.C over a temperature range of 2 to $60^{\circ} \mathrm{C}$. For the purpose of these experiments, the temperature was maintained at $26 \cdot 0^{\circ} \pm 0.2$ deg. C.

For the immunochemical studies, disk membranes from 50 retinas were collected and placed in a $1: 1$ volume of antirhodopsin serum (1:10 dilution), normal rabbit serum ( $1: 10$ dilution), or $6 \%$ serum albumin ( $1: 10$ dilution). The suspension was allowed to interact for $15 \mathrm{~min}$ with occasional agitation; it was then diluted with Ringers to a total of $15 \mathrm{ml}$. and sedimented in the Lucite sedimentation cell as previously described. The disk membranes in the pellet were not washed; thus all serum proteins were present in the pellets of disk membranes treated with the 3 sera as well as, specifically, adsorbed antirhodopsin molecules in pellets treated with antirhodopsin serum.

The low-angle cumera was similar to that described by Elliot \& Worthington (1963) and was used with a Jarrell-Ash microfocus $\mathrm{X}$-ray unit (Ni filtered $\mathrm{CuK} \alpha$ radiation). A narrow line-source of $\mathrm{X}$-rays was formed using only the first mirror in conjunction with an effective focal area of $1 \mathrm{~mm} \times 5 \mu$ on the anode. All exposures were of $2 \mathrm{hr}$ duration and the specimen-to-film distance was 5 to $8 \mathrm{~cm}$ in all experiments.

To verify the orientation of the receptor disk membranes in the pellets, the following experiments were performed. The Melinex window was removed along with the disk membrane pellet from the sedimentation cell. The cylindrical pellet was then cut along the sedimentation axis into two half-cylinder shaped portions. Such pellets, both untreated and serum treated, were then moisture-sealed (i.e. $100 \%$ humidity) in the temperature chamber at $26 \cdot 0^{\circ} \pm 0 \cdot 2 \mathrm{deg}$.C and oriented such that the $\mathrm{X}$-ray beam incident was normal to the sedimentation axis and the line focus was parallel to the plane of the Melinex to which the disk membrane pellet was attached.

Microdensitometer tracings (Joyce-Loebl \& Co., Gateshead) were made of all low-angle $\mathrm{X}$-ray diffraction patterns. 


\section{Results}

\section{(a) Electron microscopy}

Electron micrographs of negatively-stained retinal receptor disk membrane preparations showed aggregations of disk-like membranous structures (Plate I). These disk-like membranes are about $5 \mu$ in diameter and are only slightly lobated. Furthermore, direct negative staining of suspensions containing predominantly retinal rod outer segments (obtained by gently shaking an intact retina in phosphotungstate), as judged by light microscope observation under red light, also showed identical disk-like membranous structures. Ultracentrifugal pellets of these disk membranes sectioned parallel to the sedimentation axis (Plate II) show the stacked arrangement of the disk membranes along the sedimentation axis of these pellets as previously described (Blasie et al., 1965). Following glutaraldehyde fixation, it is clear that disk membranes in our preparations are present in a vesicular double-membrane form. Thus, we believe that these disk-like membranous structures in our density gradient preparations are most likely the intact disks of the frog retinal receptors or the intact lobes of retinal rod disks.

Plate III is an electron micrograph of a negatively-stained disk membrane obtained at sufficient initial magnification to resolve the $\sim \mathbf{4 0} \AA$ diameter particles of the disk membrane as previously reported (Blasie et al., 1965). A reasonably large region of background is shown and it is relatively structureless compared with the disk membrane. Plate IV is a higher magnification of Plate III. The $\mathbf{4 0 ~ \AA ~ d i a m e t e r ~ n o n - p o l a r ~}$ core of the particle is clearly shown. Plate $V$ is an optical transform obtained directly from a Kodalith print of Plate IV at identical magnification. The first-order series of reflections arising from a square array of the particles appearing in Plate IV is apparent. The unit cell side is $\sim 70 \AA$. Optical transforms obtained from masks of the electron micrographs provided essentially the same information with regards to unit cell symmetry and size (for a transform of a mask made from an electron micrograph of untreated negatively-stained disk membranes, see Blasie et al., 1965).

\section{(b) Low-angle $X$-ray diffraction}

It has been shown (Blasie et al., 1965) that the ordering of the disk membranes in the ultracentrifugal pellets is such that the planes of the disk membranes are normal to the sedimentation axis. When the $X$-ray beam is incident along the sedimentation axis, low-angle $X$-ray diffraction is recorded from electron density contrast within the planes of the individual disk membranes. These patterns are cylindrically symmetric as they are invariant to rotation of the pellet about the sedimentation axis. Low-angle $\mathrm{X}$-ray diffraction patterns recorded with the beam incident normal to the sedimentation axis and the line focus parallel to the plane of the Melinex onto which the disk membranes were sedimented showed a one-dimensional lamellar repeat, the disk-to-disk repeat, which was similar to our data (unpublished) on intact retinal rods.

Low-angle X-ray diffraction patterns were obtained with the X-ray beam incident along the sedimentation axis and also normal to the sedimentation axis, from each specimen. The first exposure took two hours while the second exposure took about 15 minutes. The latter patterns were obtained mainly to check that the orientation of the disk membranes was such that the planes of the disk membranes were oriented normal to the sedimentation axis that they gave a lamellar repeat. The untreated 


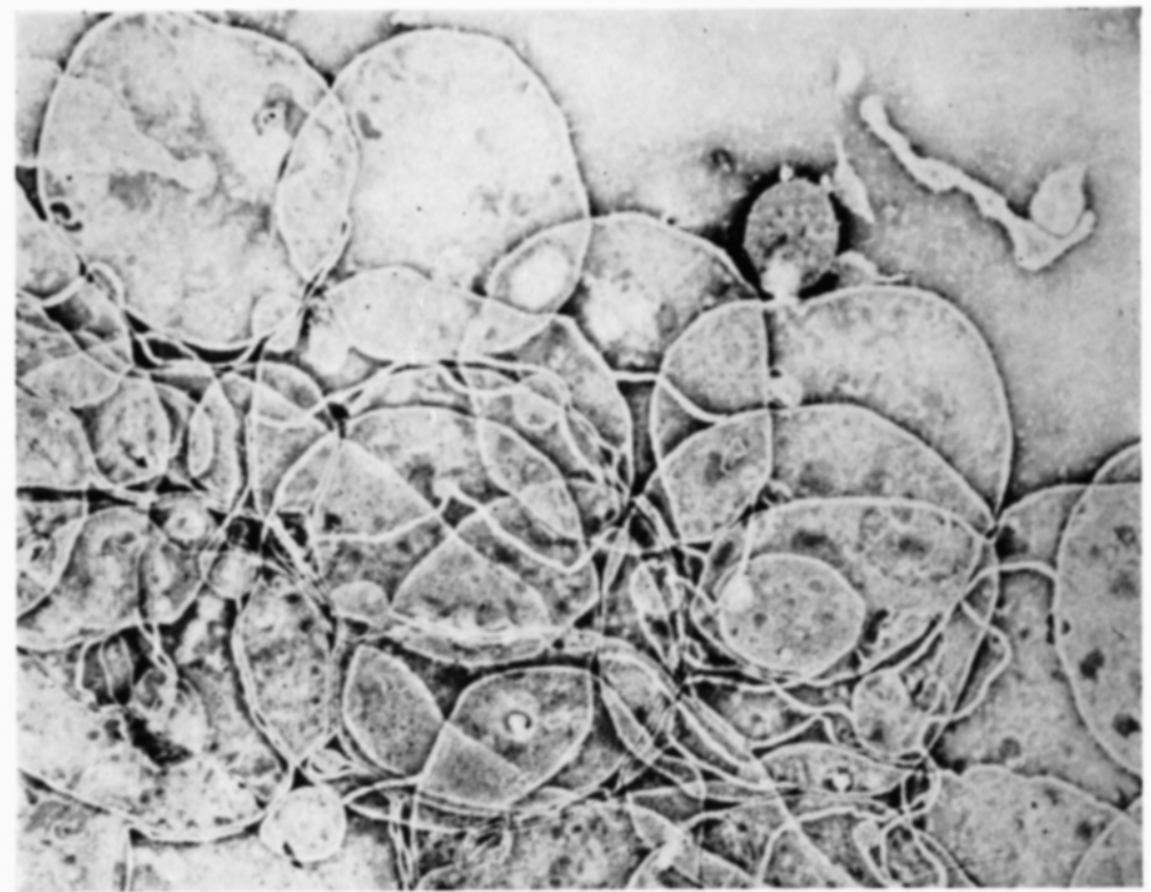

PIATE I. Frog petinal receptor disk mombranes nogatively stained with phosphotungstate. $x \sim 10,000)$.

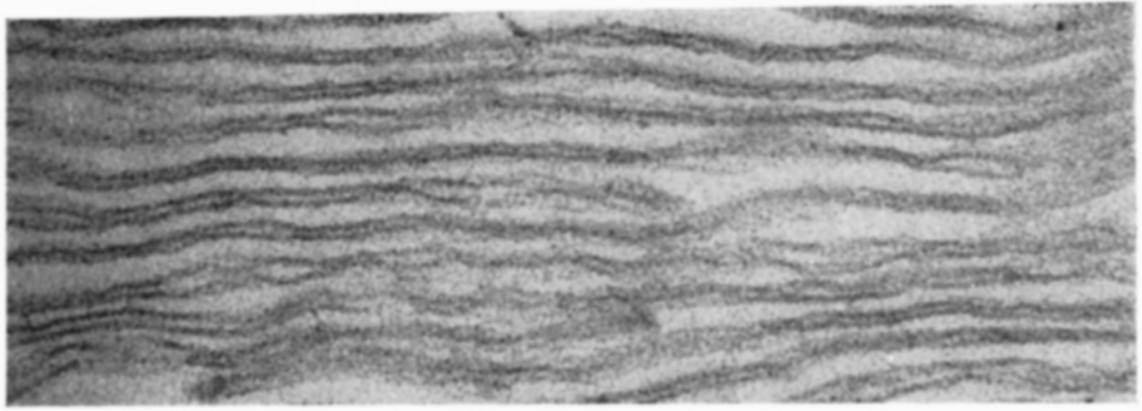

Plate II. Ultracentrifugal pellet of disk membranes fixed with glutaraldehyde and sectioned parallel to the sedimentation axis. Note the vesicular double-membrane form of the disk structure. $\times \sim 100,000$. 


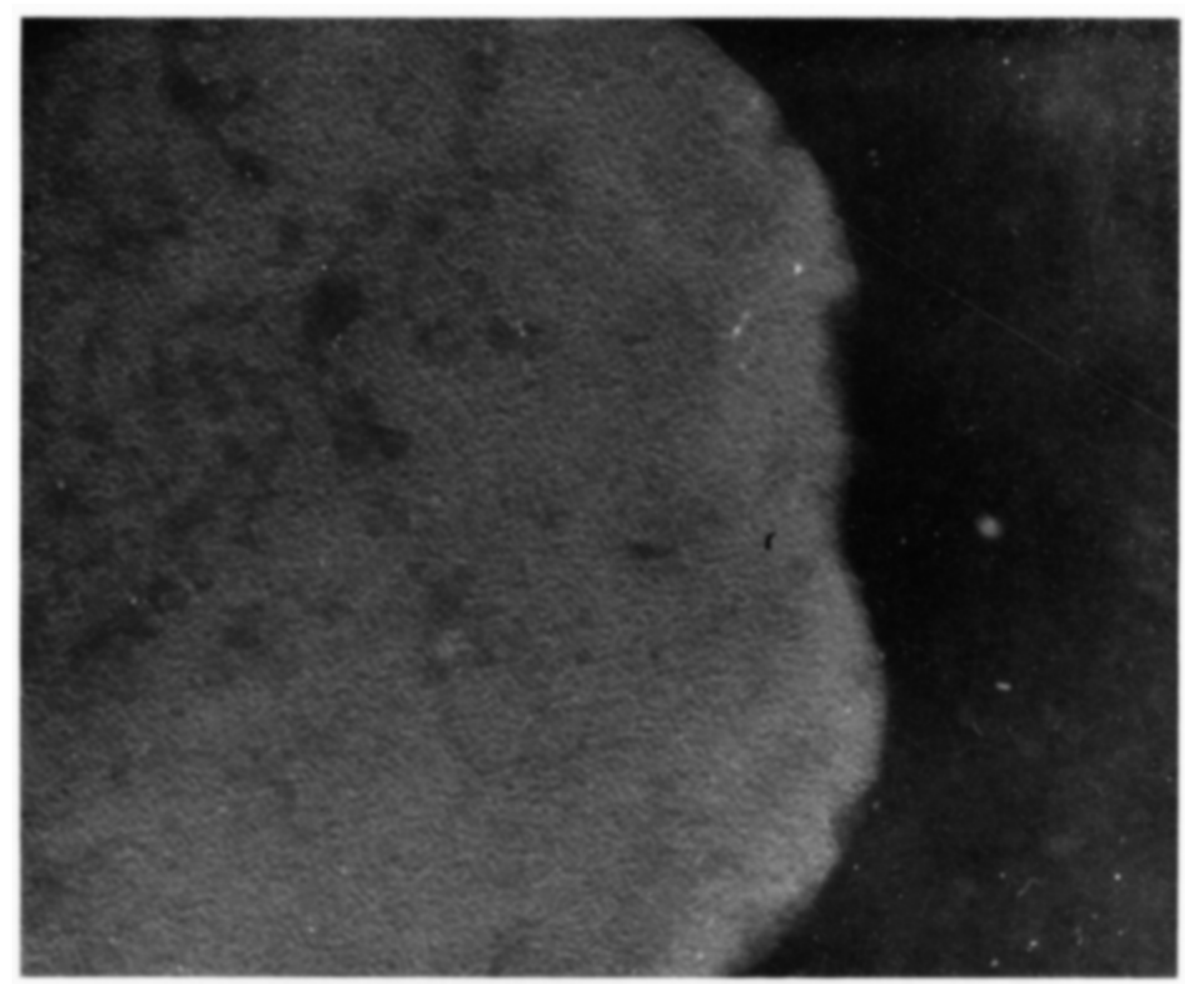

Plate III
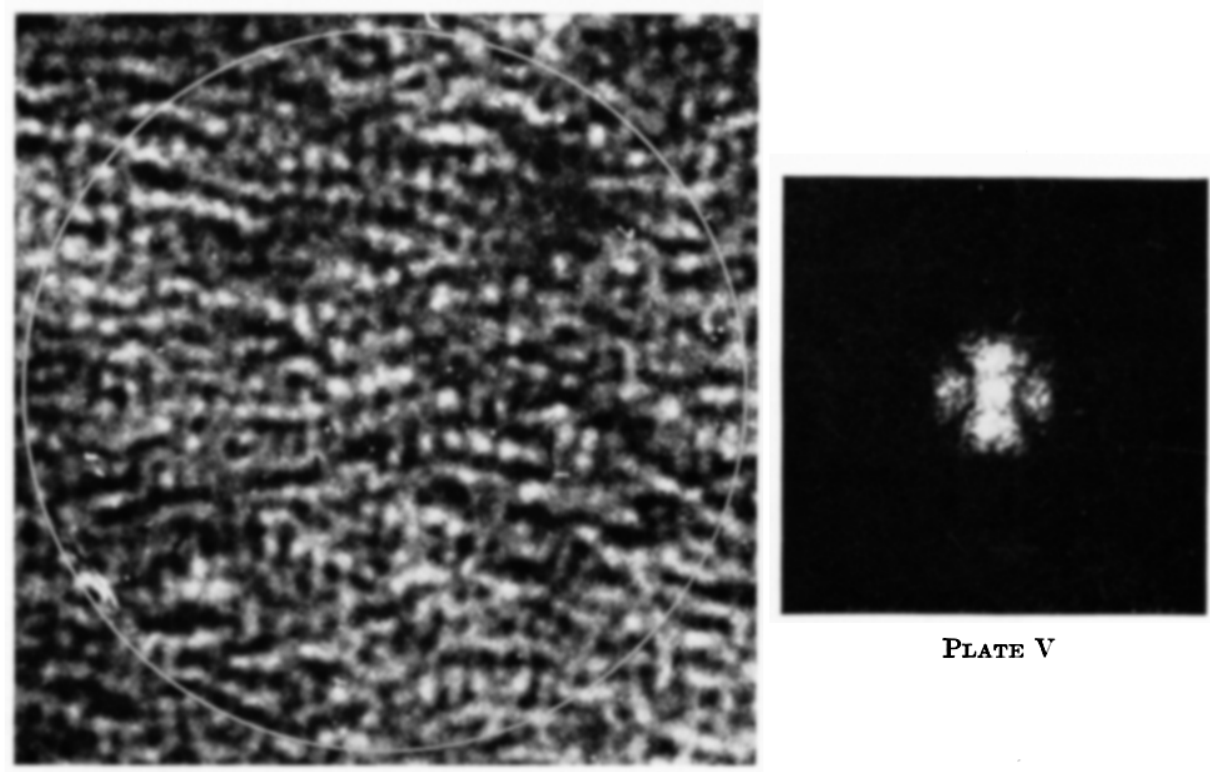

Plate V

\section{Plate IV}

Plate III. Disk membrane negatively stained with phosphotungstate. The background is relatively "structureless" compared with the disk membrane itself. $\times \sim 110,000$.

Plate IV. Higher magnification of the disk membrane shown in Plate III. The particles are $\sim 40 \AA$ in diameter. This diameter very likely represents the non-polar core of the particle inasmuch as phosphotungstate would be expected to penetrate the hydrophillie regions of the disk membrane. $\times \sim 430,000$.

Plate V. Optical transform of Plate IV. These are the $(10),(01),(10)$ and $(0 \overline{1})$ reflections representative of a planar square array of the $\sim 40 \AA$ diameter particles seen in Plate IV. The unit cell side is $\sim 70 \AA$. 

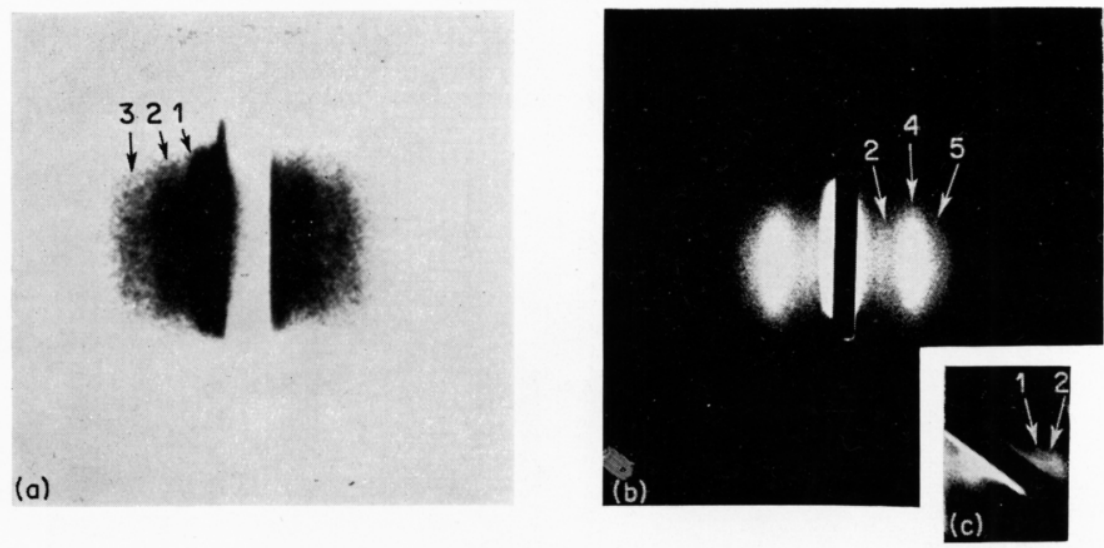

P'LATF VI. (a) Low-angle X-ray diffraction pattern obtained from moisture-sealed (1000" humidity) ultracentrifugal pellet of untreated disk membranes with the ineident beam normal to the sedimentation axis with the line focus parallel to the plane of the Melinex onto which the risk membranes were sedimented. Three orders of a $300 \AA$ disk-to-disk repeat were recorded.

(b) Low-angle X-ray pattern obtained from moisture-sealed pellet of disk membranes treated with antirhodopsin serum prior to sedimentation. The specimen-beam orientation was identical to that in (a). The second, fourth and fifth orders of a $380 \mathrm{~A}$ disk-to-disk repeat can be seen.

(c) Rotation of the above specimen $30^{\circ}$ about an axis normal to the redimentation axis revealed the first order of the 380 ) $\AA$ disk-to-disk repeat. 


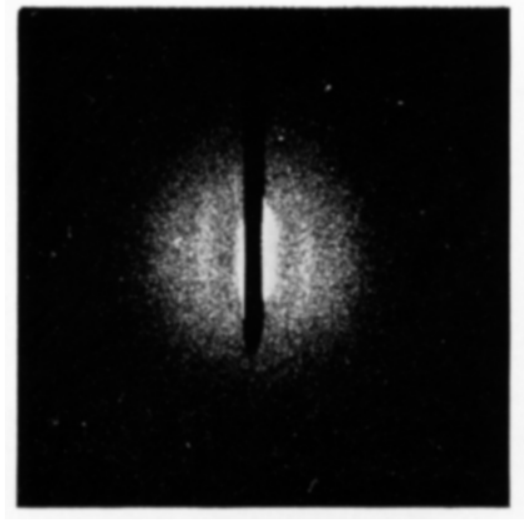

(a)

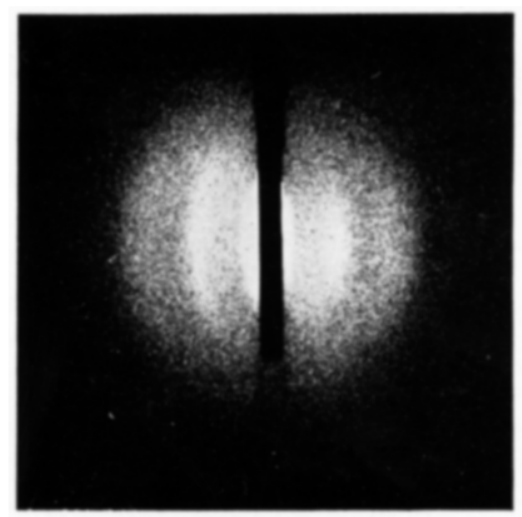

(c)

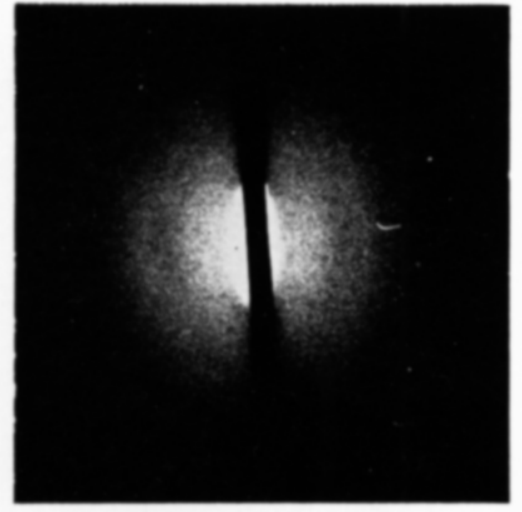

(b)

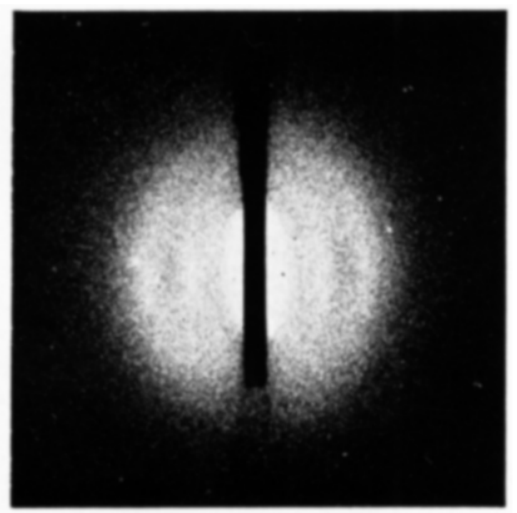

(d)

PLATe VII. (a) to (d) were obtained from completely wet specimens at $26^{\circ} \pm 0.2$ deg.C with the beam inoident along the sodimentation axis. These patterns were invariant under rotation of the specimens about the sedimentation axis. This together with the above description of Plate VI(a) to' (c) would indicate that the beam incident was normal to the planes of the disk mem. branes for this specimen-beam orientation.

(a) Wet untreated disk membranes.

(b) Wet disk membranes which had been treated with serum albumin before sedimentation.

(c) Wet disk membranes which had been treated with normal rabbit serum before sedimentation.

(d) Wet disk membranes which had been treated with antirhodopsin serum before sedimentation.

The Bragg spacings for the reflections recorded in the above patterns are given in Table 1 . 


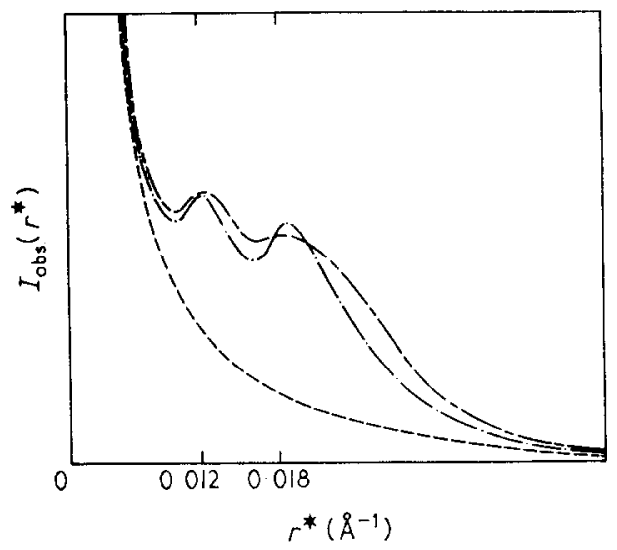

Fig. 1. Microdensitometer tracings of the patterns of Plate VII(a) and (b). Relative intensity $\left(I_{\text {obs }}\left(r^{*}\right)\right)$ is plotted as a function of the reciprocal co-ordinate $r^{*}$. - - - Untreated, $26^{\circ} \pm 0.2$ deg. $\mathrm{C} ;-\cdots$, antirhodopsin serum treated, $26^{\circ} \pm 0 \cdot 2$ deg.C.

disk membrane preparations gave three orders of a lamellar disk-to-disk repeat of about $300 \AA$ (Plate VI(a)). The disk membrane preparations treated with serum gave four to six orders of a lamellar disk-to-disk repeat of 280 to $380 \AA$. Plate VI(b) shows the lamellar disk-to-disk repeat of antirhodopsin serum treated disk membranes: the second, fourth and fifth orders of a $380 \AA$ repeat are apparent. Tilting the specimen $30^{\circ}$ about an axis normal to the sedimentation axis revealed the first order as shown in the inset Plate VI(c). An analysis of these patterns will not be presented at this time.

We describe in detail the low-angle patterns obtained with the beam incident along the sedimentation axis. In each case, the disk membrane preparations were fully hydrated, i.e. they were wet, for the duration of the exposure. The low-angle patterns and their respective densitometer tracings from the untreated and serum trealed wet disk membrane preparations are shown in Plate VII and Figure 1. The results are summarized in Table $1: d$ is the Bragg spacing, the subscript 1 and 2 refer to the first and second diffraction maxima, $I$ refers to the diffracted intensity, that is, $I\left(d_{1}\right)$ denotes the relative intensity of the first maxima.

A Fourier analysis of the low-angle X-ray diffraction data leads to a description of both the 40 to $50 \AA$ particles of the untreated disk membranes and the antirhodopsin molecules adsorbed to antirhodopsin serum treated disk membranes in terms of a planar radial distribution function. For the theory and methods of calculation, see Results section (d) of this paper and the following paper (Blasie \& Worthington, 1969). For the purposes of this paper the planar radial distribution function may be interpreted as follows: the area under a peak in the distribution function represents the time-averaged number of particles occurring within an annular ring centered about some arbitrary particle; the distance from the origin to the peak concerned is the average radius of the annular ring; the half-width or shape of such a peak is directly related to the inter-particle potential involved; the position of the maximum (as measured by the radial distance $r(\AA)$ ) of the distribution function is the most probable center-to-center distance between any particle within that annular ring and the arbitrary particle about which the annular ring is 


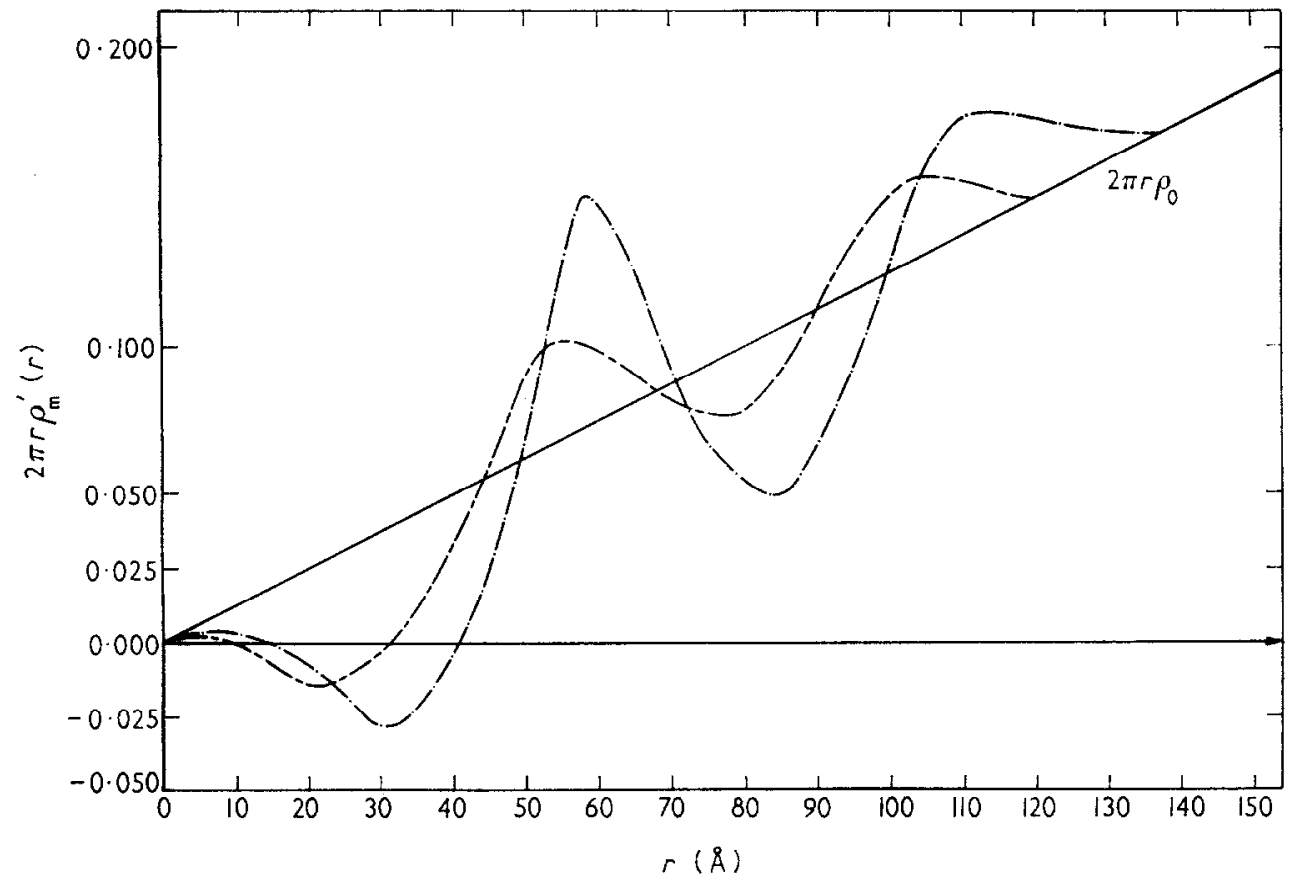

Fig. 2. Planar radial distribution functions for the 40 to $50 \AA$ particles of the untreated disk membranes at $26^{\circ} \pm 0.2 \mathrm{deg} . \mathrm{C}$ and for the specifically adsorbed cylindrical antirhodopsin molecules of the antirhodopsin serum treated disk membranes at $26^{\circ} \pm 0.2 \mathrm{deg} . \mathrm{C}$. - - Untreated, $26^{\circ} \pm 0 \cdot 2$ deg. C.; - - ; antirhodopsin serum treated, $26^{\circ} \pm 0 \cdot 2 \mathrm{deg} . \mathrm{C}$.

centered. The first maxima in the two-dimensional radial distribution function which occurs for $r$ greater than the particle diameter is referred to as the first nearest neighbor peak and the area under that peak is the time-averaged number of first nearest neighbors.

The planar radial distribution functions for the 40 to $50 \AA$ particles of the untreated disk membranes and for the antirhodopsin molecules adsorbed to the antirhodopsin serum treated disk membranes are shown in Figure 2. We note the extremely small shift in the position of the first nearest neighbor peak $(56 \AA \rightarrow 58 \AA$ ) and the small change in the number of first nearest neighbors $(3.3 \rightarrow 3.4)$ at $26.0^{\circ}$ $\pm 0.2 \mathrm{deg} . \mathrm{C}$ of the antirhodopsin molecules adsorbed to the antirhodopsin serum treated disk membranes compared to that of the 40 to $50 \AA$ particles of the untreated disk membranes. We further note the considerably sharpened distribution of the first

TABLe 1

\begin{tabular}{lcccc}
\hline $\begin{array}{c}\text { Treatment of the } \\
\text { wet disk membranes }\end{array}$ & $\begin{array}{c}d_{1} \\
(\AA)\end{array}$ & $\begin{array}{c}d_{2} \\
(\AA)\end{array}$ & $d_{1}: d_{2}$ & $I\left(d_{1}\right): I\left(d_{2}\right)$ \\
\hline Untreated & 83 & 55 & $1 \cdot 50$ & $5: 4$ \\
Antirhodopsin serum treated & 80 & 53 & $1 \cdot 52$ & $2: 3$ \\
Normal rabbit serum treated & 89 & 44 & $2 \cdot 02$ & \\
Serum albumin treated & 91 & 46 & $1 \cdot 98$ & \\
\hline
\end{tabular}


nearest neighbors of the antirhodopsin molecules as compared to the 40 to $50 \AA$ particles.

\section{(c) Interpretation of the electron microscopy}

In an earlier paper (Blasie et al., 1965) we showed that low-angle X-ray diffraction patterns obtained from dried ultracentrifugal pellets of phosphotungstate stained disk membranes (beam incident along the sedimentation axis) arise from a planar square array of particles in the stained disk membranes with a unit cell side of $\sim 70 \AA$. The relative intensities of the (10) and (11) reflections are consistent with a spherical particle whose diameter is 40 to $50 \AA$. We take this observation as independent evidence for the existence of the square array of $\sim 40 \AA$ diameter particles seen in Plate IV.

Upon drying, the two diffracted intensity maxima arising from wet pellets of untreated disk membranes (beam incident along the sedimentation axis) shown in Plate VII(a) and Figure 1 shift in a continuous fashion finally becoming the (10) and (11) reflections of a planar square array of particles with a unit cell side of $\sim 70 \AA$. The relative intensities of these two reflections are consistent with a spherical particle whose diameter is 40 to $50 \AA$ (Blasie, 1968). Thus, it is likely that the $\sim 40 \AA$ diameter particles seen in Plate IV are the same particles giving rise to the observed low-angle $\mathrm{X}$-ray diffraction arising from the wet pellets of untreated disk membranes.

\section{(d) Interpretation of the low-angle $X$-ray diffraction}

We first discuss the low-angle X-ray diffraction data, with the X-ray beam incident along the sedimentation axis, obtained from untreated and antirhodopsin serum treated wet disk membrane preparations. From Table 1 it is evident that the Bragg spacings of the two diffraction maxima are nearly identical for each of the two cases, but the ratio of intensities is quite different and the shapes of the two maxima are sharper for the serum treated disk membranes.

In order to interpret the data from the antirhodopsin serum treated disk membranes, we need to estimate the origin of the independent X-ray scattering. We have shown that antirhodopsin molecules are adsorbed onto the serum treated disk membranes under identical conditions of preparation (Dewey et al., 1969). The 40 to $50 \AA$ particle, if protein, has a density of 1.3 to $1.4 \mathrm{~g} / \mathrm{cc}$ and hence a molecular weight of 26,000 to 55,000 . The particle, if lipid, has a density of about $0.95 \mathrm{~g} / \mathrm{cc}$ and hence a micellar weight of 19,000 to 37,000 . The molecular weight of the antirhodopsin molecule is 160,000 to 170,000 . Thus, the independent scattering from the antirhodopsin molecule is 11 to 40 times that of a 40 to $50 \AA$ protein molecule and 22 to 80 times that of a 40 to $50 \AA$ spherical lipid micelle. Hence, for a qualitative analysis of the antirhodopsin serum treated disk membranes, we can safely assume that the $\mathrm{X}$-ray scattering arises only from the antirhodopsin molecules adsorbed to the disk membranes.

The ratio of the Bragg spacings is the same for both the untreated and antirhodopsin serum treated disk membranes (see Table 1). Therefore it follows, in a qualitative sense that the antirhodopsin molecules adsorbed to the disk membranes have the same ordering as the 40 to $50 \AA$ particles of the untreated disk membranes. Since the antirhodopsin molecules are known to bind specifically to the photopigment molecules of the disk membranes (Dewey et al., 1969), these arguments suggest that the 40 to $50 \AA$ particles are indeed the photopigment molecules of the receptor disk membranes and that these photopigment molecules bind antirhodopsin molecules 
in a one-to-one fashion. The difference in the ratio of the intensities in these two cases follows from the difference in the Fourier transforms of the 40 to $50 \AA$ particles and the antirhodopsin molecules, i.e. their respective scattering factors. We show from our radial distribution function studies (Blasie \& Worthington, 1969) that the shape of the 40 to $50 \AA$ particle is approximately spherical and actually 44 to $46 \AA$ in diameter while the antirhodopsin molecule is nearly cylindrical and $32 \AA$ in diameter, in agreement with the $X$-ray determination of shape of $\gamma$-globulin carried out in solution (Kratky, Porod, Sekora \& Paletta, 1955).

The low-angle $X$-ray data from the normal rabbit serum and albumin treated disk membranes is similar as can be seen in Table 1 . We find that the effect of normal rabbit serum on the diffraction pattern obtained from wet disk membranes can be duplicated by treatment with albumin alone. This is reasonable as normal rabbit serum contains a high relative percentage of albumin.

The molecular weight of albumin is known to be about 68,000 so that independent $\mathrm{X}$-ray scattering from albumin molecules non-specifically adsorbed to albumin treated disk membranes and the 40 to $50 \AA$ particles of those disk membranes is of comparable intensity. Our failure to observe diffraction from albumin treated disk membranes representative of that obtained from the 40 to $50 \AA$ particles of untreated disk membranes (as determined by the Bragg spacing ratios) is indicative of the following: (1) the albumin molecules non-specifically adsorb to a component of the disk membrane unrelated to the 40 to $50 \AA$ particles and so disrupt the previous ordering of the particles giving rise to a different diffraction pattern, the albumin and the 40 to $50 \AA$ particles scattering independently; (2) the albumin molecules non-specifically bind to the 40 to $50 \AA$ particles disrupting the previous ordering of the particles giving rise to a different diffraction pattern with the albumin-40 to $50 \AA$ particle complex scattering as a single unit. In any case, the ordering of the 40 to $50 \AA$ particles of the untreated disk membranes is disrupted by non-specific adsorption of albumin to the membranes. Apparently the ordering of the 40 to $50 \AA$ particles in the disk membrane is easily altered by weak interaction with albumin (weak relative to the interaction with antirhodopsin molecules since albumin can be washed off the disk membranes with phosphate buffered saline while the antirhodopsin cannot (Dewey et al., 1969)). This effect on the ordering of these particles is discussed in the following paper (Blasie \& Worthington, 1969).

If our interpretations of the low-angle patterns are correct, the 40 to $50 \AA$ particle is not a spherical lipid micelle. The dimensions of globular particles seen in electron micrographs of some biological membranes (Sjöstrand \& Elfvin, 1964; Nilsson, 1964, 1965; Blasie et al., 1965) are comparable to those of spherical lipid micelles observed in electron micrographs of macromolecular lipid complexes (Lucy \& Glauert; 1964) and to those of spherical lipid micelles occurring in the cubic phase of phospholipidwater systems (Luzzati \& Reiss-Husson, 1966). On the basis of dimensional comparison alone, one may only suggest that the globular particles might be spherical lipid micelles. Thus the evidence for the existence of spherical lipid micelles in biological membranes is indirect. However, it is of interest that the cubic phase of certain phospholipid-water systems is only one of many phases accessible to that system. For example, the hexagonal and lamellar phases are also accessible. Phase transitions occur readily as a function of lipid concentration and temperature. We have recorded low-angle patterns from the 40 to $50 \AA$ particles of the isolated disk membranes (in which the lipid concentration is presumably constant) over a range of temperatures 
$\left(4^{\circ}\right.$ to $\left.50^{\circ} \mathrm{C}\right)$ Blasie \& Worthington, 1969$)$. It is unlikely that spherical lipid micelles would be stable over such a temperature range. Furthermore, an analysis of the radial distribution function allows various possible scattering factors for the particles to be tested (for details, see Blasie \& Worthington, 1969). In this manner, one can show that a 40 to $50 \AA$ particle with a cross-sectional electron density characteristic of a globular protein is consistent with the $\mathrm{X}$-ray diffraction data whereas a particle with a cross-sectional electron density characteristic of a spherical lipid micelle is not. Thus, the possibility that the 40 to $50 \AA$ particles of the disk membrane are spherical lipid micelles is quite remote.

The planar radial distribution functions for the 40 to $50 \AA$ particles of the untreated disk membranes and for the antirhodopsin molecules of the antirhodopsin serum treated disk membranes are now compared. The functions were calculated using the best possible scattering factors for the particles involved, which are shown (Blasie \& Worthington, 1969) to be based on a 46 to $48 \AA$ "soft sphere" for the 40 to $50 \AA$ particles of the untreated disk membranes and a "hard cylinder" $32 \AA$ in diameter for the antirhodopsin molecules adsorbed to the antirhodopsin serum treated disk membranes. The positions of the first nearest neighbor peaks and the number of the tirst nearest neighbors are identical to within $3 \%$ on the time average. Assuming that the observed X-ray scattering arises almost solely from the antirhodopsin molecules adsorbed to the antirhodopsin serum treated disk membranes, we conclude that the antirhodopsin molecules adsorb to the surface of the disk membrane with a two-dimensional order identical to that of the 40 to $50 \AA$ particles within the disk membrane. Since the antirhodopsin molecules specifically bind photopigment, the 40 to $50 \AA$ particles of the receptor disk membrane are indeed the photopigment molecules of these membranes and in view of the identical number of first nearest neighbors for the two cases, they bind the antirhodopsin molecules in a one-to-one fashion. The sharpening of the first nearest neighbor peak for the antirhodopsin serum treated disk membranes is presumably due to increased nearest neighbor interaction along the lateral surfaces of the adjacent cylindrical antirhodopsin molecules. In contrast, spherical photopigment molecules in untreated disk membrane have considcrably less surface area available for nearest neighbor interaction. Thus, a quantitative analysis of such low-angle diffraction data tends to establish the 40 to $50 \AA$ particles of the disk membranes as the photopigment molecules of these membranes.

\section{(e) Correlation of the electron microscopy and the low-angle $X$-ray diffraction}

We present evidence in Results section (d) of this paper that the particles seen in our electron micrographs (Plates III and IV) are likely to be the same particles which give rise to the observed low-angle $X$-ray diffraction from wet pellets of untreated disk membranes with the beam incident along the sedimentation axis. The low-angle $\mathrm{X}$-ray diffraction experiments employing the antirhodopsin immune serum identify these 40 to $50 \AA$ diameter particles in the wet receptor disk membranes as the photopigment molecules. Hence, we believe that the $\sim \mathbf{4 0} \AA$ diameter particles seen in our electron micrographs of negatively-stained disk membranes are the photopigment molecules occurring in these membranes. Inasmuch as the phosphotungstate would be expected to penetrate the hydrophillic regions of the disk membrane, the $\sim 40 \AA$ diameter likely represents the non-polar core of the photopigment molecule (see also, Blasie \& Worthington, 1969). 
This work was supported by United States Public Heath Service grants AM-05197, NB-07199 and GM-09796.

\section{REFERENCES}

Blasie, J. K. (1968). Doctoral Thesis, University of Michigan, Ann Arbor.

Blasie, J. K., Dewey, M. M., Blaurock, A. E. \& Worthington, C. R. (1965). J. Mol. Biol. 14, 143.

Blasie, J. K. \& Worthington, C. R. (1969). J. Mol. Biol. 39, 417.

Dewey, M. M., Blasie, J. K., Davis, P. K. \& Barr, L. (1969). J. Mol. Biol. 39, 395.

Elliot, A. \& Worthington, C. R. (1963). J. Ultrastructure Res. 9, 166.

Kratky, O. G., Porod, A., Sekora \& Paletta, B. (1955). J. Polymer Sci. 16, 163.

Lucy, J. A. \& Glauert, A. M. (1964). J. Mol. Biol. 8, 727.

Luzzati, V. \& Reiss-Husson, F. (1966). Nature, $210,1351$.

Nilsson, S. E. G. (1964). J. Ultrastructure Res. 11, 581.

Nilsson, S. E. G. (1965). J. Ultrastmucture Res. 12, 207.

Sjöstrand, F. S. (1963). Mechanisms of Demyelination, ed. by A. S. Rose \& C. M. Pearson, p.l. New York : MeGraw-Hill.

Sjöstrand, F. S. \& Elfvin, L. G. (1964). J. Ultrastructure Res. 10, 263. 